\title{
Estudo randomizado para avaliação da dieta hipolipídica nos sintomas digestivos no pós-operatório imediato da colecistectomia por videolaparoscopia
}

\section{Randomized study for assessment of hypolipidic diet in digestive symptoms immediately following laparoscopic cholecystectomy}

Hunaldo Lima de Menezes, tCBC-Al'; Pérola Averbug Fireman²; Vicentina Esteves Wanderley33; Ângela Maria Moreira Canuto de Menconçä; Raisa Karla de Azevedo Bispo ${ }^{4}$; Melissa Ramos Reis ${ }^{4}$

\section{R E S U M O}

\begin{abstract}
Objetivo: Validar a necessidade da prescrição da dieta hipolipídica na prevenção ou redução dos sintomas dispépticos no período pós-operatório de pacientes submetidos à colecistectomia por videolaparoscopia. Métodos: Foram selecionados 40 pacientes, distribuídos em dois grupos, isentos de doenças hepáticas, pancreáticas, litíase da via biliar, gastrite, úlcera, diabetes e dislipidemia. Foi realizado anamnese alimentar, identificação de dispepsias antes do aparecimento da colelitíase e orientações sobre a conduta alimentar no pós-operatório (normal ou hipolipídica). Foi utilizado o teste chi-square e a correlação de Pearson, considerando pd"0,05 como significância estatística. Resultados: Comparando-se os dois grupos de pacientes sem dispepsias no pré-operatório, observou-se que no grupo I, sete pacientes (63,6\%) permaneceram assintomáticos e no grupo II, quatro $(66,7 \%)$. No grupo I, em quatro $(36,4 \%)$ houve aparecimento de sintomas e no grupo II, em dois $(33,3 \%)$, logo $p=0,684$. Correlacionando-se os dois grupos dispépticos no pré-operatório, observou-se que houve permanência, aparecimento ou desaparecimento dos sintomas no pósoperatório, sendo $p=0,114$. Conclusão: Não houve repercussão significativa da dieta hipolipídica na prevenção dos sintomas dispépticos, principalmente nos pacientes assintomáticos no pré-operatório. Sendo assim, não há necessidade em se orientar uma dieta hipolipídica. De modo que, cabe ao cirurgião avaliar cada paciente individualmente e ajustar a dieta às necessidades do paciente e às condições clínicas associadas.
\end{abstract}

Descritores: Colecistectomia. Dispepsia. Sinais e sintomas disgetórios. Gorduras na dieta. Terapia Nutricional.

\section{INTRODUÇÃO}

A colecistectomia videolaparoscópica é o método de escolha no tratamento da colelitíase ${ }^{1,2}$. O objetivo da cirurgia é o alívio dos sintomas e o tratamento e/ou prevenção de complicações ${ }^{1,3,4}$. Contudo, em cerca de $10 \%$ a $50 \%$ dos pacientes submetidos à colecistectomia, os sintomas persistem ou são desenvolvidos novos sintomas ${ }^{1,3}$. A persistência ou aparecimento de sintomas após o procedimento cirúrgico é chamada de síndrome póscolecistectomia². Estes sintomas são geralmente inespecíficos e leves e constituem em flatulência, náuseas, eructação e indigestão $0^{1,3}$

A causa mais comum de síndrome póscolecistectomia são as desordens extra-biliares, tais como doença ulcerosa péptica, doença do refluxo gastroesofagiano e síndrome do intestino irritável. Apenas em uma minoria dos casos os sintomas se devem a doença do trato biliar ${ }^{1,3}$.

Não existe na atualidade motivo válido para restringir a dieta de um paciente submetido à colecistectomia em seu pós-operatório, já que a vesícula não é um órgão de produção de bile, e sim, de armazenamento ${ }^{5}$. Entretanto, muitos consideram uma medida de cautela limitar o consumo de lipídeos nas primeiras semanas após o procedimento cirúrgico, acreditando que isto reduziria a chance de desenvolvimento de sintomas típicos da síndrome póscolecistectomia 5 . Isto gera uma controvérsia entre os cirurgiões com relação à indicação ou não de uma dieta hipolipídica no pós-operatório desses pacientes. Até hoje, não existem trabalhos científicos suficientes que mostrem a eficácia ou não da prescrição da dieta hipolipídica após a colecistectomia.

Trabalho realizado nos Serviços de Cirurgia Geral e Gastroenterológica e de Nutrição do Hospital Memorial Arthur Ramos, Maceió, Alagoas, Brasil.

1. Professor da Disciplina de Sistema Digestório da Faculdade de Medicina da Universidade Federal de Alagoas, Maceió-AL-BR; 2. Coordenador do Serviço de Nutrição do Hospital Memorial Arthur Ramos, Maceió-AL-BR; 3. Professora da Disciplina de Sistema Digestório da Faculdade de Medicina da Universidade Federal de Alagoas, Maceió-AL-BR; 4. Acadêmica da Faculdade de Medicina da Universidade Federal de Alagoas, Maceió-AL-BR. 
Com o intuito de identificar e orientar o melhor o planejamento alimentar dos pacientes, o presente trabaIho pretendeu avaliar a necessidade da prescrição dessa dieta na prevenção ou redução dos sintomas gastrointestinais durante o período pós-operatório em pacientes submetidos à colecistectomia por videolaparoscopia.

\section{MÉTODOS}

Foi realizado um ensaio clínico randomizado em que foram selecionados 97 pacientes com colelitíase que seriam submetidos à colecistectomia por videolaparoscopia, atendidos nos mbulatórios de Cirurgia Geral, Gastroenterologia e Emergência no Hospital Memorial Arthur Ramos, em Maceió/AL. O diagnóstico foi obtido após exames clínico, laboratorial e de imagem.

Foram incluídos no estudo pacientes de ambos os sexos com quadro de colelitíase, que no pré-operatório não apresentassem doenças hepatopancreáticas associadas, após serem submetidos aos seguintes exames: ultrassonografia do abdômen total; endoscopia digestiva alta; análise bioquímica (AST, ALT, Gama-GT, fosfatase alcalina, bilirrubina direta, indireta e total, amilase pancreática, lipase pancreática, glicose, colesterol total, LDL e HDL-colesterol e triglicerídeos).

Foram excluídos da pesquisa portadores de gastrite, úlcera gástrica ou duodenal, hepatopatias, pancreatopatias, litíase da via biliar, diabetes e dislipidemias.

Os pacientes foram avaliados através de formulários contendo os resultados obtidos dos exames pré-operatórios a fim de descartar hepatopatias, pancreatopatias, gastrite, úlcera e litíase da via biliar, que associados à colelitíase pudessem confundir a avaliação dos sintomas do pós-operatório, que não são inerentes ao trabalho em questão.

Destes, foram selecionados 40 pacientes que correspondem a $41,2 \%$ da amostra pesquisada. Foi realizada randomização em bloco através de uma tabela de números randômicos, constituindo-se dois grupos de 20 pacientes: O grupo I que seguiria uma dieta hipolipídica no pós-operatório, e o grupo II uma dieta normal. Foi realizada uma anamnese sucinta nos dois grupos direcionada à ingesta de alimentos ricos em lipídeos, objetivando uma melhor orientação no pós-operatório e, ao mesmo tempo, não estimulando a introdução deste tipo de alimento em pessoas do grupo II que não tinham o hábito anteriormente, pois poderiam surgir sintomas no pós-operatório em pacientes que possuem algum tipo de intolerância ou dispepsia antes do aparecimento da colelitíase.

Assim, após a avaliação dietética foi estabelecida a dieta recomendada após a colecistectomia. Foram estabelecidos e tabelados os sintomas do pré-operatório e do pós-operatório, para facilitar a construção da análise do trabalho e seus resultados.
Os pacientes receberam, na ocasião do internamento hospitalar, um formulário contendo o planejamento alimentar para ser seguido no pós-operatório e orientados a retornar em 15 dias para coleta de dados.

$\mathrm{Na}$ análise estatística, empregou-se o teste chisquare e a correlação de Pearson, considerando $p<0,05$ como significância estatística.

Este trabalho foi aprovado pelo Comitê de Ética em Pesquisa do Hospital Memorial Arthur Ramos, segundo parecer de $n^{\circ}$ 001/2011, de 02/06/2011.

\section{RESULTADOS}

Entre os 40 pacientes submetidos à colecistectomia que obedeceram aos critérios de inclusão, havia 32 do sexo feminino (80\%) e oito do sexo masculino (20\%). A idade variou entre 18 e 76 anos, com média de 47 anos.

No período pré-operatório dos dois grupos estudados, observamos que 17 (42,5\%) eram assintomáticos e 23 (57,5\%) sintomáticos.

Dentre os pacientes sintomáticos o que mais predominou no pré-operatório foram constipação e flatulência. Estes apareceram sozinhos, juntos ou associados a outros sintomas (Figura 1).

Após a divisão dos grupos, observamos que no grupo que recebeu dieta hipolipídica, 11 (55\%) pacientes eram assintomáticos e nove (45\%) eram sintomáticos no pré-operatório.

No pós-operatório dos pacientes assintomáticos, sete (35\%) permaneceram assintomáticos, quatro (20\%) passaram a apresentar sintomas e dos pacientes sintomáticos dois (10\%) passaram a ser assintomáticos e sete (35\%) permaneceram sintomáticos.

Do grupo que recebeu dieta normal, seis (30\%) eram assintomáticos e 14 (70\%) sintomáticos no pré-operatório.

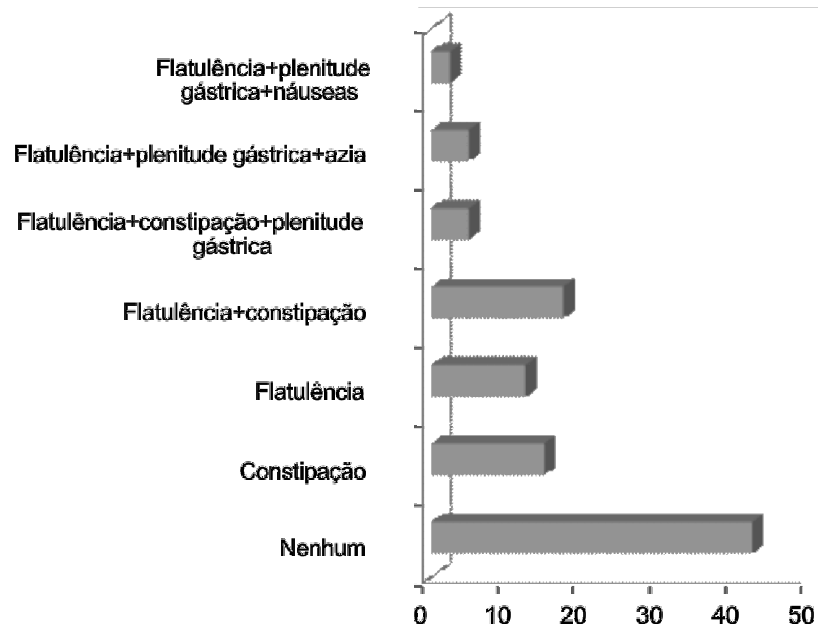

Figura 1 - Sintomas no pré-operatório de ambos os grupos. 
No pós-operatório dos pacientes assintomáticos, quatro (20\%) permaneceram assintomáticos, dois (10\%) passaram a apresentar sintomas, já os pacientes sintomáticos três (15\%) passaram a ser assintomáticos e 11 (55\%) permaneceram sintomáticos.

Considerando os pacientes assintomáticos no préoperatório de ambos os grupos, observamos que: no grupo I (dieta hipolipídica), dos 11 pacientes, sete (63,6\%) permaneceram sem sintomas digestivos no pós-operatório e em apenas quatro $(36,4 \%)$ houve aparecimentos de sintomas. No grupo II (dieta normal), dos seis pacientes, quatro $(66,7 \%)$ permaneceram sem sintomas digestivos no pósoperatório e em dois $(33,3 \%)$ houve aparecimento de sintomas (Tabela 1):

A análise estatística mostrou, através do teste qui-quadrado ( $\mathrm{p}$ £ 0,05) como significância estatística, que não há diferença significativa entre os pacientes assintomáticos no pré-operatório que seguiram a dieta hipolipídica e os da dieta normal na prevenção ou redução dos sintomas dispépticos durante o período pós-operatório de 15 dias, pois $p>0,05$.

Considerando os pacientes sintomáticos no préoperatório de ambos os grupos, observamos que dos nove pacientes no grupo I, em dois $(22,2 \%)$ houve desaparecimento dos sintomas digestivos no pós-operatório, seis $(66,7 \%)$ permaneceram com os mesmos sintomas e em um $(11,1 \%)$ permaneceu um sintoma e houve uma troca de sintomas, ou seja, desapareceu um, porém apareceu outro diferente.

No grupo II dos 14 pacientes, em três $(21,4 \%)$ houve desaparecimento dos sintomas digestivos no pós- operatório, em dois $(14,3 \%)$ houve troca de sintomas, e dois $(14,3 \%)$ permaneceram com os mesmos sintomas, em um $(7,1 \%)$ houve a adição de um sintoma ao do quadro pré-operatório, em outros quatro $(28,6 \%)$ permaneceram alguns e desapareceram outros e em dois $(14,3 \%)$ permaneceram alguns e houve troca de sintomas (Tabela 2).

A análise estatística evidenciou, através do teste qui-quadrado $(p £ 0,05)$, que não há diferença significativa entre os pacientes sintomáticos no pré-operatório que seguiram a dieta hipolipídica e os da dieta normal na prevenção ou redução dos sintomas digestivos durante o período pós-operatório de 15 dias, pois p>0,05.

\section{DISCUSSÃO}

Na nossa amostragem, a colelitíase foi quatro vezes mais frequente no sexo feminino, o que está de acordo com a literatura, que refere uma frequência de três a quatro vezes maior no sexo feminino ${ }^{2,4,6-9}$ As influências hormonais parecem ser os fatores responsáveis, como o uso de anticoncepcionais na mulher fértil e estrogênio na pós-menopausa, que aumentam o potencial litogênico da bile, além da vulnerabilidade psicológica ${ }^{7,9}$. A média de idade encontrada também corresponde com a média encontrada na literatura ${ }^{4,7,9}$.

A maioria dos pacientes colecistectomizados apresentou sintomas variados no pós-operatório ${ }^{1,10}$. Os encontrados no trabalho foram constipação, flatulência, náuseas e plenitude gástrica, o que está de acordo com a

Tabela 1 - Comparação dos sintomas no pós-operatório dos dois grupos de pacientes assintomáticos no pré-operatório.

\begin{tabular}{lrrrrr}
\hline Sintomas & \multicolumn{3}{c}{ Hipolipídica } & \multicolumn{2}{c}{ Normal } \\
& $(\mathrm{n})$ & $(\%)$ & $(\mathrm{n})$ & $(\%)$ \\
\hline Permaneceram assintomáticos & 7 & 63,6 & 4 & 66,7 \\
Aparecimento de sintomas & 4 & 36,4 & 2 & 33,3 \\
Total & 11 & 100,0 & 6 & 100,0 \\
\hline
\end{tabular}

Teste qui-quadrado de Pearson com correção de continuidade de Yates $p=0,6847$

Tabela 2 - Comparação dos sintomas no pós-operatório dos dois grupos de pacientes sintomáticos no pré-operatório.

\begin{tabular}{lcccc}
\hline Sintomas & \multicolumn{2}{c}{ Hipolipídica } & \multicolumn{2}{c}{ Normal } \\
& $(\mathrm{n})$ & $(\%)$ & $(\mathrm{n})$ & $(\%)$ \\
\hline Desaparecimento & 2 & 22,2 & 3 & 21,4 \\
Troca de sintomas & - & - & 2 & 14,3 \\
Permanência dos sintomas & 6 & 66,7 & 2 & 14,3 \\
Permanência e adição de outros sintomas & - & - & 1 & 7,1 \\
Permanência de alguns e desaparecimento de outros & - & - & 4 & 28,6 \\
Permanência e troca de sintomas & 1 & 11,1 & 2 & 14,3 \\
Total & 9 & 100,0 & 14 & 100,0 \\
\hline
\end{tabular}

Teste qui-quadrado de Pearson sem correção de continuidade de Yates $p=0,1146$ 
literatura ${ }^{7-9,11}$. Um sintoma frequente relatado na literatura, mas que não foi encontrado no trabalho foi a intolerância à ingestão de alimentos gordurosos ${ }^{7,8}$.

Como observamos, no pré-operatório de ambos os grupos os sintomas que predominaram foram flatulência e constipação e ambos permaneceram no pós-operatório na maioria dos pacientes. A literatura também aponta esses sintomas como de não significativa melhora após a colecistectomia ${ }^{7,8}$.

Nos dois grupos, independente da dieta preconizada, mais da metade dos pacientes assintomáticos permaneceu assintomático no pós-operatório, sendo 63,63\% naqueles que seguiram a dieta hipolipídica e $66,66 \%$ nos que seguiram a dieta normal, evidenciando uma diferença insignificante.

O restante dos pacientes assintomáticos no préoperatório, em 36,36\% houve aparecimento de sintomas naqueles com dieta hipolipídica e 33,32\% com dieta normal, também não significativo.

Considerando-se os pacientes sintomáticos no préoperatório de ambos os grupos, houve desaparecimento, aparecimento ou permanência de sintomas no pós-operatório.

O uso da dieta hipolipídica no pós-operatório após a colecistectomia é controverso na literatura e entre os cirurgiões, pois apesar de não se ter documentada melhora significativa dos sintomas com esta dieta, muitos médicos a prescrevem e, quando não o fazem, muitos pacientes continuam com a dieta após o procedimento cirúrgico por receio de seu consumo, visto que alimentos ricos em gordura estão relacionados à intolerância e ao aparecimento dos sintomas no período préoperatório ${ }^{5,7}$.

Nos dois grupos houve desaparecimento dos sintomas dispépticos, como a plenitude gástrica, náuseas e azia, mas houve a persistência dos sintomas colônicos, como flatulência e constipação. Essa variabilidade de sintomas presentes no pós-operatório também é controversa na literatura, pois uma parte dos autores diz que os sintomas que mais persistem após a operação são os dispépticos, mas também já foram encontrados a persistência dos sintomas colônicos e desaparecimento dos dispépticos ${ }^{7,11}$.

Nos pacientes que já apresentavam dispepsia no pré-operatório e que parecem não estar correlacionada à colelitíase, e nem tampouco com a colecistectomia, mantiveram-se com os mesmos sintomas após a operação, essa permanência dos sintomas pode está ligada a vulnerabilidade psicológica, como aponta a literatura ${ }^{4,7,11-13}$.
Vários são os fatores envolvidos com a fisiopatologia e a etiopatogenia da síndrome póscolecistectomia ${ }^{12}$. Alguns destes sintomas podem ser causados por distúrbios orgânicos, como formação de cálculos no ducto biliar comum após a operação, úlcera péptica e assim por adiante. Entretanto, a origem exata dos sintomas não é clara ${ }^{12}$.

Acredita-se que alguns sintomas podem ter origem em outras doenças do trato digestivo, que, após a presença de litíase biliar à investigação, foram erroneamente associados à litíase. Outros sintomas podem ser causados por distúrbios diretamente relacionados à colecistectomia, como a estenose biliar, os cálculos retidos, a estenose ou a discinesia do esficter de Oddi, a diarreia induzida pelos sais biliares e o ducto cístico longo remanescente ${ }^{3,5,10,13,14}$. Por outro lado, os opioides e outros fármacos utilizados em doses elevadas na anestesia geral, como o citrato de fentanila e o alfentanil, derivados da morfina, têm como efeitos colaterais náuseas e vômitos, além de reduzir a motilidade gastrointestinal, provocando constipação ${ }^{1,6}$.

Foi constatado que a persistência dos sintomas possui várias causas, sendo que $50 \%$ dos pacientes possuem algum tipo de desordem orgânica pancreatobiliar ou no sistema gastrointestinal. Ao restante dos pacientes podem ser atribuídas causas psicossomáticas ${ }^{13}$.

A etiologia da continuação dos sintomas após a colecistectomia é multifatorial, sendo ainda alvo de estudos futuros que devem ser voltados para as mudanças fisiológicas que ocorrem após o procedimento, visando além da prevenção, a melhor seleção dos pacientes recomendados à colecistectomia eletiva ${ }^{12}$.

Os resultados obtidos no presente estudo demonstram que não houve repercussão significativa da dieta hipolipídica na prevenção ou redução dos sintomas dispépticos no pós-operatório imediato da colecistectomia por videolaparoscopia, principalmente naqueles assintomáticos no pré-operatório. Assim, a prescrição de uma dieta com baixo teor de gordura depende da linha que o cirurgião prefere seguir e do perfil do paciente e suas condições clínicas associadas, pois alguns preferem continuar a dieta hipolípidica que é feita no pré-operatório após a colecistectomia, sentindo-se melhor por causa de fatores psicológicos.

Assim recomendamos que o cirurgião avalie individualmente cada paciente e ajuste a dieta às suas necessidades e às condições clínicas associadas, em algumas ocasiões é necessário que o paciente seja encaminhado ao serviço de Nutrição. 


\section{A B S S T R A C T}

Objective: To validate the need for prescribing low-fat diet in the prevention or reduction of dyspeptic symptoms in the postoperative period in patients undergoing laparoscopic cholecystectomy. Methods: We selected 40 patients, free of liver or pancreatic disease, biliary gallstones, gastritis, ulcer, diabetes and dyslipidemia, who were divided into two groups. We conducted dietary anamnesis, identification of dyspepsia before the onset of cholelithiasis and guidance on appropriate postoperatively feeding (normal or lowfat). We used the chi-square test and Pearson correlation for statistical assessment, considering $p d^{\prime \prime} 0.05$ as significant. Results: When comparing the two groups of patients without preoperative dyspepsia, it was observed that in group / seven patients (63.6\%) were asymptomatic and in group II, four (66.7\%). In group I, four (36.4\%) had onset of symptoms and in group II, two (33.3\%), $p=$ 0.684. When correlating the two groups with preoperative dyspeptic symptoms, it was observed that there was permanence, appearance or disappearance of symptoms postoperatively, $p=0.114$. Conclusion: There was no significant effect of low-fat diet in the prevention of gastrointestinal symptoms, especially in preoperatively asymptomatic patients. Thus, there is no need of a lowfat diet. So, it is up to the surgeon to evaluate each patient individually and adjust the diet to his/her needs and clinical conditions.

Key words: Cholecystectomy. Dyspepsia. Signs and symptoms, digestive. Dietary fats. Nutrition therapy.

\section{REFERENCIAS}

1. Coelho JCU, Nassif AE, Campos ACL. Vesícula Residual. Rev Col Bras Cir. 2002;29(6):367-9.

2. Csendes Juhasz A, Csendes G P, Rojas C J, Sánchez R M. Resultados de la colescistectomía a 10 años plazo. Rev méd Chile. 2000;128(12):1309-12

3. Salim MT, Cutait R. Complicações da cirurgia videolaparoscópica no tratamento de doenças da vesícula e vias biliares. $A B C D$ arq bras cir dig. 2008;21(4):153-7.

4. Gutiérrez de la Peña C, Garcia Molina F, Méaquez Platero R, Domínguez-Adame E, Medina Díez J. Persistencia de síntomas en el postoperatorio de la colecistectomía laparoscópica por colelitiasis sintomática. Cir Esp. 2001;70(4):187-90.

5. Zúniga GA. Revisión bibliográfica: el síndrome postcolecistectomia. Rev méd Hondureña. 1986;54(3):237-40.

6. Fujii $Y$. The utility of antiemetics in the prevention and treatment of postoperative nausea and vomiting in patients scheduled for laparoscopic cholecystectomy. Curr Pharm Des. 2005;11(24):317383.

7. Luman W, Adams WH, Nixon SN, Mcintyre IM, Hamer-Hodges D, Wilson $G$, et al. Incidence of persistent symptoms after laparoscopic cholecystectomy: a prospective study. Gut. 1996;39(6):863-6.

8. Gui GP, Cheruvu CV, West N, Sivaniah K, Fiennes AG. Is cholecystectomy effective treatment for symptomatic gallstones? Clinical outcome after long-term follow-up. Ann R Coll Surg Engl. 1998;80(1):25-32.

9. Mjåland $\mathrm{O}, \mathrm{H}$ Ægevold $\mathrm{HE}$, Buanes $\mathrm{T}$. Standard preoperative assessment can improve outcome after cholecystectomy. Eur J Surg. 2000;166(2):129-35.
10. Lum YW, House MG, Hayanga AJ, Schweitzer M Postcholecystectomy syndrome in the laparoscopic era. J Laparoendosc Adv Surg Tech A. 2006;16(5):482-5.

11. Borly L, Anderson IB, Bardram L, Christensen E, Sehested A, Kehlet $\mathrm{H}$, et al. Preoperative prediction model of outcome after cholecystectomy for symptomatic gallstones. Scand J Gastroenterol. 1999;34(11):1144-52.

12. Middelfart HV, Kristensen JU, Laursen CN, Qvist N, H/Ejgaard L, Funch-Jensen $\mathrm{P}$, et al. Pain and dyspepsia after elective and acute cholecystectomy. Scand J Gastroenterol. 1998:33(1):10-4.

13. Schofer JM. Biliary causes of postcholecystectomy syndrome. Emerg Med. 2010;39(4):406-10.

14. Jaunoo SS, Mohandas S, Almond LM. Postcholecystectomy syndrome (PCS). Int J Surg. 2010;8(1):15-7.

Recebido em 28/06/2012

Aceito para publicação em 16/08/2012

Conflito de interesse: nenhum

Fonte de financiamento: nenhuma

\section{Como citar este artigo:}

Menezes HL, Fireman PA, Wanderley VE, Mencoça AMMC, Bispo RKA. Avaliação da dieta hipolipídica nos sintomas digestivos no pósoperatório imediato da colecistectomia por videolaparoscopia. Rev Col Bras Cir. [periódico na Internet] 2013;40(3). Disponível em URL: http://www.scielo.br/rcbc

\section{Endereço para correspondência:}

Hunaldo Lima de Menezes

E-mail: hunaldomenezes@arthurramos.com.br 